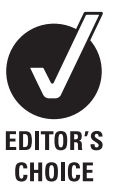

(

${ }^{1}$ Department of Medical History and Ethics, Vilnius University,

Vilnius, Lithuania

${ }^{2}$ Department of Medical Ethics,

Lund University, Lund, Sweden

${ }^{3}$ Riga Stradins University, Riga,

Latvia

${ }^{4}$ Department of Philosophy and

Social Sciences, Kaunas

University of Medicine, Kaunas, Lithuania

${ }^{5}$ Department of Humanities,

Riga Stradins University, Riga,

Latvia

${ }^{6}$ Department of Public Health,

University of Tartu, Tartu,

Estonia

${ }^{7}$ Bioethics Program, Union

Graduate College - Mount Sinai

School of Medicine,

Schenectady, New York, USA

Correspondence to

Eugenijus Gefenas, Department

of Medical History and Ethics,

Vilnius University, M.K.Ciurlionio

St. 21/27, Vilnius LT-03101,

Lithuania;

eugenijus.gefenas@mf.vu.lt

Received 14 December 2009

Revised 8 April 2010

Accepted 12 April 2010

\title{
Non-equivalent stringency of ethical review in the Baltic States: a sign of a systematic problem in Europe?
}

\author{
E Gefenas, ${ }^{1}$ V Dranseika, ${ }^{1}$ A Cekanauskaite, ${ }^{1}$ K Hug, ${ }^{2}$ S Mezinska, ${ }^{3}$ E Peicius, ${ }^{4}$ \\ V Silis, ${ }^{5}$ A Soosaar, ${ }^{6}$ M Strosberg ${ }^{7}$
}

\section{ABSTRACT}

We analyse the system of ethical review of human research in the Baltic States by introducing the principle of equivalent stringency of ethical review, that is, research projects imposing equal risks and inconveniences on research participants should be subjected to equally stringent review procedures. We examine several examples of non-equivalence or asymmetry in the system of ethical review of human research: (1) the asymmetry between rather strict regulations of clinical drug trials and relatively weaker regulations of other types of clinical biomedical research and (2) gaps in ethical review in the area of nonbiomedical human research where some sensitive research projects are not reviewed by research ethics committees at all. We conclude that non-equivalent stringency of ethical review is at least partly linked to the differences in scope and binding character of various international legal instruments that have been shaping the system of ethical review in the Baltic States. Therefore, the Baltic example could also serve as an object lesson to other European countries which might be experiencing similar problems.

In some countries, variations of procedural stringency of ethical review may reflect an underdeveloped infrastructure of research ethics committees (RECs) where their approval is seen simply as a formality to comply with the requirements of international partners. ${ }^{1}$ Such RECs function as a "rubber stamp" to legitimise research and become, in the words of Richard Ashcroft, a "figleaf" covering institutions not able to perform their mission of protecting the interests of research subjects. ${ }^{2}$ However, much more complex issues of varying stringency of ethical review stem from some systematic asymmetries in the research regulations. We analyse asymmetries or cases of non-equivalent stringency of ethical review in the Baltic States of Estonia, Latvia and Lithuania that recently attempted to build within a rather short time span a research ethics review system based on international instruments and regulations. Since the early attempts in the late 1980s, when first RECs were established as a result of local initiatives in particular research institutions, ethical review of human research in these countries has undergone significant change influenced by a series of legal and institutional developments. Estonia has chosen the simplest system of ethical review with two RECs based in two main medical research institutions of the country. Lithuania has developed a two-tier system of ethical review that includes the National Bioethics Committee issuing approvals for multicentre biomedical research studies and two regional RECs. Latvia followed the most diverse path establishing nine different RECs that vary in their institutional affiliation and types of protocols reviewed. Detailed description of RECs in the Baltic States can be found in the study of Dranseika et al. . $^{3}$ However, despite the attempts to establish a balanced system of ethical review and mentioned differences between institutional structures of ethical review in the Baltic States, some common problems could still be observed. In this paper, we analyse three different types of non-equivalence or asymmetry where research of comparable risk and burden fail to achieve similar procedural scrutiny of ethical review.

\section{EQUIVALENCE OF THE PROCEDURES OF ETHICAL REVIEW}

The system of ethical review of human research is based on the idea that research projects imposing equal or similar amount of risks and inconveniences on research participants should be subjected to equally or similarly stringent review procedures. We call it the principle of equivalent stringency of ethical review. This principle can be applied to types of research or to particular research projects. David Hunter recently formulated a version of this principle applicable to particular research projects (which he categorised as "proportional ethics review") in the following way: "the level of ethical review and scrutiny given to a research project ought to reflect the level of ethical risk represented by that project" (p. 241). ${ }^{4}$ However, he was critical of the principle on grounds that even if it is intuitively appealing, it is impracticable. We agree with Hunter's conclusions; therefore, in this paper, we will be concerned with this principle only as it relates to the types of research.

To sum up, the principle of equivalent stringency justifies varying degrees of procedural stringency of ethical review. The riskier and more burdensome a particular type of human research is, the stricter the requirements of ethical review should be. By the same token, if two types of research are similar in risks and burdens, there is a good reason to apply procedures of similar stringency.

The principle is intuitively appealing and may also serve as a device to diagnose inconsistencies inherent in the system. First, gaps of ethical review where some types of human research involving 
risks are excluded from ethical review altogether indicate that system is not well-balanced and that research participants are not sufficiently protected. Since the main mission of RECs is protection of welfare and interests of research participants, gaps are worrisome.

The more complex cases of non-equivalent stringency are asymmetries between procedural stringency of ethical review of different types of human research that involve similar level of risks, for example, the asymmetry between review of clinical drug trials and other types of clinical research. In this case, the equivalence might not be a problem from the point of view of research participants because they need only to be assured that research in which they participate will be subjected to sufficient ethical scrutiny. On the other hand, for other stakeholders involved in human research, non-equivalence may be problematic. Anything above sufficiency level places unnecessary burden on researchers, sponsors (stricter review may delay approval and increase ethical review fees) and society as a whole. Larger ethics review infrastructure may divert resources from potentially more useful endeavours. The development of science and medicine and the economic benefits produced by pharmaceutical industry are the other values that should also not be ignored. The principle of equivalent stringency of ethical review helps recognise the interests of various stakeholders involved in research.

In addition, non-equivalence-randomness between levels of depth of review-produces the appearance of incoherence, potentially damaging to society's confidence in the system. Adherence to the principle of equivalence should help to create a more coherent and predictable system.

In this paper, we do not aim to define sufficient levels of ethical scrutiny for different types of research nor do we intend to provide an analysis of "sufficiency". However, we can stipulate a formal requirement that if a certain level of ethical scrutiny is regarded as sufficient (insufficient) by research participants (and RECs since one of their functions is to protect interests of the research participants) for a certain type of research, then the same level of scrutiny should be considered sufficient (insufficient) for some other type of research which is equal in risks to the first one. It is important to note, however, that cases of non-equivalence can be identified even if one does not have a way to tell what level of procedural stringency would be adequate for a particular type of research. Our goal is quite modest: we do not aim to solve the problem of procedural stringency in relation to every single research project but rather try to show that there are systematic asymmetries related to the types of research as they are usually defined in international normative documents defining the procedures of ethical review. For the purposes of this paper, we will also limit our discussion to the "procedural" framework of ethical review and will not deal with the substantive ethical issues such as how should the risks and inconveniences imposed on research participants correlate with the ability to give an informed consent.

\section{CRITERIA OF PROCEDURAL STRINGENCY OF ETHICAL REVIEW}

The assessment of procedural stringency of ethical review is based on the following questions:

1. What is the scope of ethical review and what research projects are exempted from the review procedure? What is the possibility of expedited review? For example, human behavioural science research projects that do not exceed a socalled minimal risk threshold to research participants are reviewed by expedited procedures in Canada and the USA. ${ }^{5} 6$

2. What are the institutions (RECs of different levels and institutions other than RECs) involved in the review process?
3. How comprehensive is this process of ethical review? For example, is it limited to one-time approval or does it also include on-going monitoring and other additional safeguards?

4. Finally, does the system have the power to make ethical review legally binding or does it only have the power of recommendation? This is an important distinction because legally binding review provides for clear sanctions in case of non-compliance with the law. Legal regulations are also applicable country-wide to all the relevant research practices and institutions rather than just to a single institution as may be the case with the policy of a particular REC established at a particular research institution.

\section{THREE CASES OF NON-EOUIVALENT STRINGENCY OF ETHICAL REVIEW \\ Clinical drug trials versus other types of clinical research}

Consider first the case of non-equivalence between rather strict regulations of clinical drug trials (CDTs) and relatively weaker regulations of other types of clinical biomedical research. For example, clinical drug trials in the Baltic States are reviewed by RECs and state drug agencies ("competent authorities" as they are called in The Clinical Trials Directive of $2001^{7}$ ), while other types of clinical research are reviewed by RECs only. In addition, RECs must be notified about any amendment of the CDT and all serious adverse events. They also must receive annual study reports. For almost all other non-drug clinical research, these safeguards are missing. In Latvia, the gap between the stringency of standards is even wider because there are no legally binding regulations for ethical review of biomedical research except for CDTs, research on medical devices (an outcome of the EU Medical Devices Directive ${ }^{8}$ ) and research on the Latvian population genetic database. 9 The situation is further complicated by the fact that Latvia has not yet ratified the Oviedo Convention. ${ }^{10}$ In Estonia, which has ratified the convention, all biomedical research projects should in principle be subjected to ethical review. However, there are no national regulations on how this should be implemented in practice (eg, what sanctions could be applied for non-compliance). Despite the aforementioned differences, in all three countries, we might in principle encounter a situation where a phase IV CDT (which is often directly beneficial to the research participants) might undergo much more stringent ethical review as compared to a randomised clinical trial of different cardio-surgical interventions or some risky studies in physiology and sport sciences involving sleep deprivation.

Another problematic area with respect to ethical review appears in areas of human research not directly linked to the biomedicial and health field. Two groups of cases may be distinguished here: non-biomedical research involving risks to psychological health and non-biomedical research involving risks to privacy (eg, disclosure of personal information to the third parties).

\section{Non-biomedical research that involves risks to psychological health}

Even if the Lithuanian system of ethical review is rather comprehensive covering all types of research related to human health, ${ }^{11}$ sensitive sociological, anthropological or psychological human research studies that do not directly fall into the area of biomedicine would not be subjected to any formalised ethical review. In fact, there are no national regulations, legally binding or otherwise, in this field of human research. Similar gaps might also be seen in Latvian and Estonian ethical review systems. For example, it is likely that studies on sexual practices, suicidal 
tendencies or child abuse, initiated by psychologists and conducted outside healthcare institutions, would bypass the ethical review procedures even if they are associated with significant risks to psychological health. Therefore, if the aforementioned studies were to be reviewed by the RECs, it would be due to the requirements of research institutions and particular RECs, scientific journals and or funding institutions, or the study would be reviewed by a special ethics board established by a professional association. For example, the Lithuanian Psychological Association has recently established an ethics committee. However, the information about the procedures of such a review and the ethics committee itself is not publicly available.

\section{Non-biomedical research that involves risks to privacy}

The lacunae similar to those described in the previous section can be identified in cases of human research projects involving risks to privacy and are carried out outside the healthcare context. This type of research is less likely to be subjected to ethical review than similar research on medical data or archived biological material. Ethical review of the latter kind of research is legally required in Lithuania ${ }^{11}$ and in some cases in Latvia (eg, research on the Latvian population genetic database ${ }^{9}$ ) and Estonia (eg, research on the Estonian population genetic database $^{12}$ and e-health databases ${ }^{13}$ ), but in Latvia and Lithuania, there are no legally binding requirements to conduct ethical review of non-biomedical research that may involve risks to privacy. This might be seen as a problem because research that deals with such sensitive data as criminal record or data on adoptions may involve threats to privacy comparable to those related to health data. It should be noted that in the EU countries, processing of sensitive personal data for research purposes is subjected to previous checking by data protection authorities following the provisions of the EU Data Protection Directive. ${ }^{14}$ However, the Directive does not specifically require the ethical review of such research projects.

The two aforementioned cases of non-biomedical research show that the requirements of ethical review of differing stringency in the Baltic States would apply to different types of human studies depending not on the differences in risks involved but on whether the research project falls within the scope of biomedicine. This does not seem to be a problem in the USA where federal research regulations define the scope of ethical review for the whole field of human research including nonbiomedical projects (103(b)). ${ }^{15}$ Similarly, the Swedish Act on the
Ethical Review of Research Involving Humans requires review of biomedical and non-biomedical research projects, where research is performed according to a method that aims to affect the subject physically or psychologically or which involves an obvious risk of physical or psychological damage to the research person. ${ }^{16}$ It would be important to check how the aforementioned lacunae are dealt with in other European countries.

\section{TYPES OF HUMAN RESEARCH ACCORDING TO INTERNATIONAL REGULATIONS}

To better understand the asymmetries and gaps of ethical review in the Baltic States mentioned above, let us briefly examine those international regulations specifically addressing ethical review of different types of human research.

For the purposes of this paper, we distinguish between several different categories of research: human research (which includes biomedical and non-biomedical studies involving human subjects), narrowly and widely defined biomedical research as well as clinical drug trials. These terms are helpful for our discussion as they define the scope of important European and international instruments and guidelines that shape ethical review procedures in the Baltic States and other European countries. The general framework informing of these definitions and corresponding international regulations is summarised in figure 1.

Clinical drug trials (or alternatively, "clinical trials on medicinal products for human use"7) represent the most strictly regulated type of human research. In Europe, the most influential document providing the uniform regulatory framework of the CDTs is The Clinical Trials Directive of 2001, a legally binding document on all EU member states. ${ }^{7}$ CDTs are an important sub-category (figure 1, field 1) within the broader field of biomedical research.

The term biomedical research includes CDTs and covers all other human research studies in the health field. It extends into a much wider field of clinical research where different diagnostic, preventive or therapeutic interventions are tested without the use of medicinal products. Biomedical research is an important term in the European context because of another legally binding European instrument, namely, The Additional Protocol on Biomedical Research to the Convention on Human Rights and Biomedicine. ${ }^{17}$ The Protocol, however, is based on a narrow definition of biomedical research as it only covers research projects "in the health field" that include "physical intervention" or "any other intervention as long as it involves a risk to the
Figure 1 Relationship between different types of human research and the international regulatory framework.

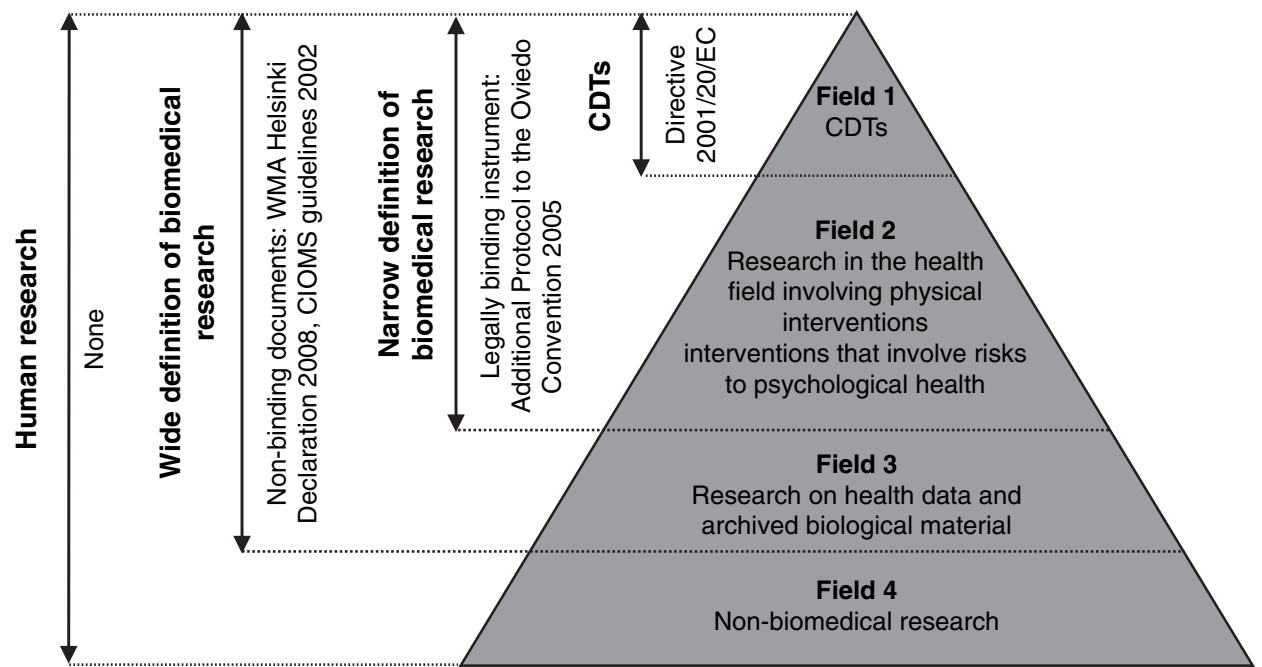


psychological health of the person concerned"17: Art. 2 (figure 1, field 2), which means that such important areas of non-clinical research as research on archived biological material as well as research on personal data do not fall within its scope.

The two aforementioned types of non-clinical biomedical research (figure 1, field 3 ) have been specifically addressed by other European documents. For example, research on archived biological material has been addressed by a Council of Europe Recommendation, ${ }^{18}$ while research on personal data has been covered by the EU Data Protection Directive. ${ }^{14}$ These fields of research are also included into the scope of such well-known international guidelines as WMA Helsinki Declaration (which covers "medical research involving human subjects, including research on identifiable human material and data"19: Para 1) and CIOMS guidelines (which covers "both medical and behavioural studies pertaining to human health" ${ }^{20}$ : Preamble). Helsinki and CIOMs guidelines employ a wide definition of biomedical research as their scope covers fields 1,2 and 3 (see figure 1). It is important to note that all the aforementioned regulations are not legally binding recommendations except for the EU Data Protection Directive. ${ }^{14}$ However, the Directive does not provide specific provisions on ethical review. Therefore, it is important to note that the field 3 of non-clinical research is in principle less strictly regulated as compared to the fields 1 and 2 (areas of narrowly defined biomedical research).

Finally, human research studies that do not fall within the field of biomedical research (non-biomedical studies) are the least regulated examples of human research on the European and international level (see the most peripheral field 4 in figure 1). There are no binding European regulations covering, for example, sociological or psychological human studies. Usually, these types of human research are left to the self-regulatory mechanisms of universities, professional organisations and scientific journals.

\section{THE SOURCES OF NON-EQUIVALENT STRINGENCY}

Having distinguished between different types of human research and having articulated criteria of procedural stringency of ethical review, we now introduce a more systematic analysis of nonequivalent stringency of ethical review in the Baltic States and make some related observations. First, there should be equivalent stringency of ethical review within the area of narrowly defined biomedical research (ie, between figure 1 fields 1 and 2)similarly stringent procedures should be applied to CDTs and other types of clinical research that involve physical intervention and risks because the general levels of risk of CDTs and other types of clinical research are similar. We assume that this asymmetry arises because The Clinical Trials Directive ${ }^{7}$ is a binding European instrument requiring adoption of the directive into the national law. All the EU Member States including the New Member States have had to harmonise their clinical drug trials legislation when joining the EU. Regulations related to all other kinds of biomedical research are much less likely to be backed by legally binding instruments. Even if ethical review of biomedical research is supposed to be brought into greater harmony by the Additional Protocol, ${ }^{17}$ so far, very few European countries have ratified the Protocol (as of September 2009, only five Council of Europe member states, none of Baltic States, have ratified it).

There should also be equivalence between certain types of biomedical and non-biomedical research because stringency of review of research involving risks to psychological health and risks to privacy should depend on the extent of risk rather than on whether that research falls within the scope of biomedical research. With regard to research involving risks to psychological health, equivalence is needed between certain types of research within figure 1 fields 4 and 2 . One should note here that ratification of the Additional Protocol ${ }^{17}$ by individual member states tends to increase this stringency gap because its scope only covers the health field.

The non-equivalence between fields 4 and 3 is of a different kind because research on personal data and archived biological material (those studies that do not fall within the confines of figure 1 field 2-narrowly defined biomedical research) is not related to the same risk of harm and inconveniences as one that involves physical or psychological intervention. However, here, the asymmetry between biomedical and non-biomedical studies also exists. Therefore, it also seems that there should be equivalence between certain divisions within figure 1 fields 3 and 4; that is, between a part of widely defined biomedical research that does not involve physical interventions and risks to psychological health and those non-biomedical studies that do not involve considerable immediate psychological risks but involve risks to privacy.

\section{ATTEMPTS TO COMPENSATE FOR NON-EOUIVALENT STRINGENCY OF ETHICAL REVIEW}

Even in the absence of national regulations that set uniform standards of ethical review in the country, the procedures of ethical review may be enforced by "softer" mechanisms or regulations-for example, policy statements/guidelines issued by RECs themselves, the requirements of different funding bodies or publishing policies of medical journals that require proof of REC approval as a condition of acceptance of a paper for publication. However, it should be noted that the policy of a particular REC may only extend to research projects carried out in that particular institution, as is the case in Estonia and Latvia.

This combination of legal and other types of regulations could provide a sufficient basis for enforcing a policy of ethical review of different types of human research especially in the countries with longer traditions of ethical review. However, at the same time, different standards of ethical review for different types of research make the effectiveness of ethical review contingent on local circumstances which in case of the transition societies might not always provide research participants with sufficient protection. Ashcroft notes that ethics committees in Eastern Europe may sometimes be established "more to satisfy foreign partners' or research sponsors' requirements, rather than to address problems of concern to patients." (p. 230) ${ }^{2}$ The problem is that the countries which have only recently established the institutions of ethical review might lack the "softer" mechanisms of regulations that ensure all types of human research being reviewed by RECs or these mechanisms might appear to be too weak to facilitate the change of practice.

\section{CONCLUDING REMARKS}

Apparently, systems of ethical review of human research in the Baltic States are not based on established and comprehensive rules covering all types of research projects. Rather, a mixture of different social regulations applies to different types of human research. This analysis revealed some systematic features of ethical review and protection of research participants by (1) showing how international and European guidelines and legal standards are being applied in societies where the tradition of institutionalised ethical review is rather recent and (2) pointing out areas of weakness in the framework of ethical review of human research on the European level. 
Variations of the binding force of the international human research regulations provide at least partial explanation of existing examples of non-equivalent stringency of ethical review. The phenomenon of asymmetry between different types of clinical research is mostly connected to the patchwork-like nature of different European instruments that regulate different types of research studies. We think that in this respect, more harmonisation is needed in Europe. In case of non-biomedical human research, "softer mechanisms" described above may at least in part compensate for the lack of more systematic provisions. However, it is important to make sure that stringency of ethical review depends not on whether research is conducted in the health field, but on the nature of risks involved.

The "diagnosis" of some examples of systematic non-equivalence suggests the need to implement a consistent system of regulations for all types of human research. However, we do not intend to claim that this problem should be solved by introducing legal regulations of ethical review of the same stringency as applied to the CDTs or that non-biomedical human research be treated in the same procedural way as biomedical research. Caution should be applied to ensure that harmonisation does not result in over-regulation, which is also possible in this field. For example, see recent public consultation paper issued by European Commission that discusses whether The Clinical Trials Directive ${ }^{7}$ has resulted in over-regulation. ${ }^{21}$ Some other, perhaps less stringent solution might be preferable. However, first it is important to recognise that current system of ethical review is susceptible to non-equivalent stringency of ethical review.

Acknowledgements The project was supported by Award Number R25TW007085 for the Fogarty International Center. The content is solely the responsibility of the authors and does not necessarily represent the official views of the Fogarty International Center or the National Institutes of Health. We also want to thank anonymous referees for valuable suggestions how to make this paper clearer.

Funding Other Funders: NIH.

Competing interests Andres Soosaar is at the University of Tartu Human Research Ethics Committee. Eugenijus Gefenas and Asta Cekanauskaite are at the Lithuanian Bioethics Committee. Eimantas Peicius is at Kaunas Regional Biomedical Research Ethics Committee.

Provenance and peer review Not commissioned; externally peer reviewed.

\section{REFERENCES}

1. Coleman C, Bouësseau MC. How do we know that research ethics committees are really working? The neglected role of outcomes assessment in research ethics review. BMC Med Ethics 2008;9:6.

2. Ashcroft RE. Commentary: Ethics Committees and Countries in Transition: a figleaf for structural violence? Br Med J 2005;331:229-30.
3. Dranseika V, Gefenas E, Cekanauskaite A, et al. 20 years of Human Research Ethics Committees in the Baltic States. Developing World Bioethics (Forthcoming).

4. Hunter D. Proportional Ethical Review and the Identification of Ethical Issues, $J$ Med Ethics 2007:33:241-5.

5. University of Ottawa. Minimal risk review and expedited review. http://www.rges uottawa.ca/ethics/minimal risk.asp (accessed Apr 2010).

6. Florida State University. Human Subjects Committee. http://www.research.fsu. edu/humansubjects (accessed Apr 2010).

7. Anon. Directive 2001/20/EC of 4 April 2001, of the European parliament and of the Council on the approximation of the laws, Regulations and administrative provisions of the Member States relating to implementation of good clinical practice in the conduct of clinical trials on medicinal products for human use. Official Journal of the European Union 2001:121:34.

8. Anon. Council Directive 93/42/EEC of 14 June 1993 concerning medical devices. Official Journal of the European Union 1993;169:1-43.

9. Saeima of the Republic of Latvia. 2002. Republic of Latvia Law on Human Genome Research, 2002. http://www.ttc.lv/export/sites/default/docs/LRTA/Likumi/ Human Genome Research_Law.doc (accessed Apr 2010).

10. Council of Europe. Convention for the Protection of Human Rights and Dignity of the Human Being with regard to the Application of Biology and Medicine: Convention on Human Rights and Biomedicine, 1997. http://conventions.coe.int/Treaty/en/ Treaties/Html/164.htm (accessed 5 Apr 2010).

11. Seimas of the Republic of Lithuania. Republic of Lithuania Law on Ethics of Biomedical Research, 2000. http://www3.lrs.lt/pls/inter3/dokpaieska.showdoc_e? p id=148740 (accessed 5 Apr 2010).

12. The Parliament of Estonia. Estonian human genes research act, 2000. http:// www. geenivaramu.ee/index.php?id=98 (accessed 5 Apr 2010).

13. The Parliament of Estonia. Estonian Health Services Organisation Act (Tervishoiuteenuste korraldamise seadus), 2001. http://www.riigiteataja.ee/ert/act. jsp?id=13202311 (accessed 5 Apr 2010)

14. Anon. Directive 95/46/EC on the protection of individuals with regard to the processing of personal data and on the free movement of such data. OJL 1995:281:31-50.

15. Department of Health and Human Services. Code of Federal Regulations. Title 46 Public Welfare. Part 46 Protection of Human Subjects, 2005. http://www.hhs.gov/ ohrp/humansubjects/guidance/45crr46.htm (accessed 5 Apr 2010).

16. Swedish Ministry of Education and Cultural Affairs, 2003. The Act concerning the Ethical Review of Research Involving Humans (Lag om etikprövning av forskning som avser människor). http://www.riksdagen.se/webbnav/index.aspx? nid $=3911$ cbet $=2003: 460$ (accessed 5 Apr 2010).

17. Council of Europe. Additional protocol to the convention on human rights and biomedicine, concerning biomedical research, 2005. http://conventions.coe.int/ treaty/en/Treaties/Html/195.htm (accessed 5 Apr 2010)

18. Council of Europe Recommendation $\operatorname{Rec}(2006) 4$ of the Committee of Ministers to member states on research on biological materials of human origin, 2006. http:// www.coe.int/t/e/legal_affairs/legal_co-peration/bioethics/texts_and_documents/ Rec 2006 4.pdf (accessed 5 Apr 2010).

19. World Medical Association. Declaration of Helsinki - Ethical Principles for Medica Research Involving Human Subjects (Helsinki: 1964; amended Tokyo: 1975; Venice: 1983; Hong Kong: 1989; Republic of South Africa: 1996; Edinburgh: 2000; Washington: 2002; Tokyo 2004; Seoul 2008). 2008. http://www.wma.net/en/ 30publications/10policies/b3/index.html (accessed Apr 2010).

20. Council for International Organizations of Medical Sciences (CIOMS). International Ethical guidelines for biomedical research involving human subjects. Geneva, Switzerland: CIOMS, 2002. http://www.cioms.ch/ frame guidelines nov 2002.htm (accessed Apr 2010).

21. European Commission. Assessment of the Functioning of the 'Clinical Trials Directive' 2001/20/EC, 2009. Public Consultation Paper. http://ec.europa.eu/ enterprise/pharmaceuticals/clinicaltrials/docs/2009_10_09_public-consultationpaper.pdf (accessed 5 Apr 2010). 